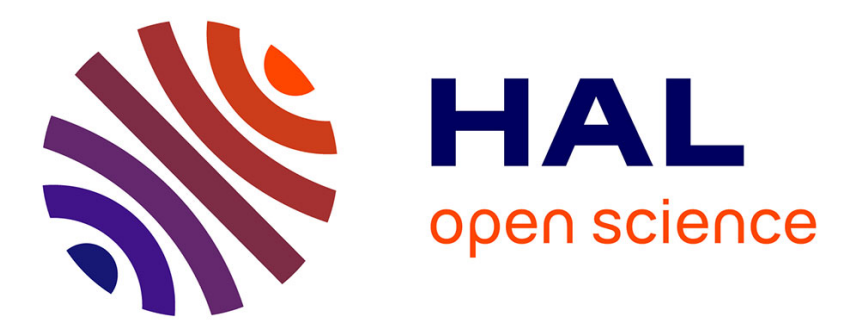

\title{
Nonlinear static response of low-aspect-ratio inverted flags subjected to a steady flow
}

\author{
Mohammad Tavallaeinejad, Michael Païdoussis, Mathias Legrand
}

\section{To cite this version:}

Mohammad Tavallaeinejad, Michael Païdoussis, Mathias Legrand. Nonlinear static response of lowaspect-ratio inverted flags subjected to a steady flow. Journal of Fluids and Structures, 2018, 83, pp.413-428. 10.1016/j.jfluidstructs.2018.09.003 . hal-01745147v2

\section{HAL Id: hal-01745147 \\ https://hal.science/hal-01745147v2}

Submitted on 19 Oct 2018

HAL is a multi-disciplinary open access archive for the deposit and dissemination of scientific research documents, whether they are published or not. The documents may come from teaching and research institutions in France or abroad, or from public or private research centers.
L'archive ouverte pluridisciplinaire HAL, est destinée au dépôt et à la diffusion de documents scientifiques de niveau recherche, publiés ou non, émanant des établissements d'enseignement et de recherche français ou étrangers, des laboratoires publics ou privés. 


\title{
Nonlinear static response of low-aspect-ratio inverted flags subjected to a steady flow
}

\author{
Mohammad Tavallaeinejad, Michael P. Païdoussis, Mathias Legrand \\ Department of Mechanical Engineering, McGill University, Montréal, Québec, Canada H3A OC3
}

\begin{abstract}
The nonlinear static response of inverted flags, otherwise known as cantilevered plates in a reverse steady axial flow, is investigated. Recent studies have shown that the stability of low-aspect-ratio inverted flags in fluid flow is different to that of wide inverted flags. The latter undergo a supercritical divergence with increasing flow velocity, while a low-aspect-ratio inverted flag buckles abruptly within a wide bistable region. This paper aims to understand the effect of aspect ratio on the stability and bifurcation characteristics of the multiple equilibria of low-aspect-ratio inverted flags. To this end, the inverted flag is idealized as a thin Euler-Bernoulli beam coupled with the time-invariant form of the fluid forces obtained from a potential flow theory. A Hamiltonian framework is employed to derive the nonlinear integro-differential equation governing the motion expressed in terms of the mid-line rotation angle of the flag along its longitudinal axis. This allows for reliable predictions even at very large deflection amplitudes. The presence of an initial curvature along the length of the flag is also accounted for in the modelling. The Galerkin modal decomposition technique along with a Newton method is employed and the fixed-points of the governing equation are found. Bifurcation diagrams of the response with respect to the flow velocity are constructed. It is shown that the system with small aspect ratio undergoes a static divergence via a subcritical pitchfork bifurcation followed by a saddle-node bifurcation. Supercritical pitchfork bifurcations are expected for sufficiently large aspect ratios. In addition, numerical simulations are performed to investigate the effect of initial curvature amplitude on the nonlinear response of the system. The numerical predictions stemming from the proposed model compare well with existing experimental measurements available in the literature. However, it should be noted that some of the reported static responses of the inverted flag might violate the assumption of potential flow theory.
\end{abstract}

Keywords inverted flag, subcritical divergence, bistability, nonlinear bending, fluid-structure interactions

\section{Introduction}

Plates interacting with flow are present in many engineering applications ranging from energy harvesters to design of micro air vehicles and autonomous vehicle propulsion. Such applications have motivated a considerable amount of research $[8,22,9,10]$. Furthermore, many researchers have employed plate-interactingwith-flow models to explain trivial everyday phenomena such as flapping of flags or motion of tree leaves in a gentle breeze [31, 27], as well as to analyse the in-flight or swimming motion of animals and explain the mechanism of snoring in humans $[38,17,3]$.

A cantilevered plate interacting with flow loses stability via a static divergance or growing amplitude of oscillations, depending on its orientation to the flow. From a design point of view, this phenomenon can be either detrimental, for structures such as paper in printing processes and aerospace systems [37, 36], or beneficial, for energy harvesters subject to flow-induced flutter [14]. Therefore, understanding the dynamical behaviour of a rectangular plate interacting with flow is of significant importance; it would provide access to accurate predictions of the fluidelastic instabilities in complex real-world fluid-structure interaction problems.

A great deal of research has been conducted on the stability of plates interacting with flow. These studies may be classified into three categories based on the configuration of the rectangular plate interacting with flow. The first configuration is a wing-type cantilevered plate with a single clamped edge parallel to the flow, which has received attention for aerospace applications. The second configuration is a cantilevered 
plate with a fixed leading edge and a free trailing edge, aligned with the flow, referred to as an flag-type configuration. Many researchers have investigated the stability of such systems theoretically $[15,11,19,17$, $35,2,33]$ and experimentally [31, 7, 41, 40, 12, 42, 29]; a thorough recent survey of the literature on these aspects is performed by Païdoussis [23]. A third configuration which has recently received attention due to potential applications in small scale flutter-based energy harvesting systems, is referred to as inverted-flag-type configuration, or inverted flag; as opposed to the flag-type configuration, the leading edge of the inverted flag is free while the the trailing one is fixed. In fact, energy harvesting inverted piezoelectric flags are shown to feature several advantages compared to the conventional piezoelectric flags [30,16,22], a such as larger amount of harvested ambient energy and lower operating range of flow velocity, which is more expected in typical ambient conditions. Furthermore, an inverted flag exhibits several characteristics distinct from the flag-type configuration, such as bifurcations, response amplitude, and sensitivity to geometrical parameters.

The present study is devoted to investigating the nonlinear dynamical behaviour of systems belonging to this third category. The number of studies in the literature on the dynamics of an inverted flag interacting with flow is rather limited and can be grouped into three general classes, i.e., experimental investigations, analytical studies using continuum expressions for the aerodynamic forces and computational fluid dynamics (CFD) investigations utilizing numerical solvers for the Navier-Stokes equations.

Starting with the first two classes, the first study in this field was conducted by Guo and Païdoussis [15], who employed an inviscid potential flow model along with a linear beam structure model to study the dynamics of plates in axial flow. They solved the perturbation pressure field in Fourier space while neglecting the wake, and predicted the onset of flutter. Kim et al. [18] revealed experimentally that the inverted flag remains stable in the trivial zero-deflection equilibrium configuration up to a critical flow velocity. Increasing the flow velocity causes the flag to oscillate and finally bend in a fully deflected configuration.

In two remarkable studies, Sader et al. [26, 27] pursued the investigations of Kim et al. [18] and conducted a set of experiments to explore the effect of the aspect ratio (i.e. the ratio of span to chord). Their experimental observations have revealed that the dynamics of the system is dramatically affected by the flag aspect ratio. In particular, they reported the existence of a bistable region, wherein low-aspect-ratio flags exhibit a buckling behaviour with a highly curved shape. Intermediate and wide flags, on the other hand, undergo an abrupt flapping motion initiated by a divergence instability. Moreover, they reported that the undeflected static equilibrium of inverted flags with smaller aspect ratios loses stability at higher critical flow velocities.

In addition to experiments, Sader and co-workers investigated the effect of the aspect ratio parameter separately in two asymptotic limits. In the first study [27], they employed a two-dimensional model based on a steady fluidelastic theory introduced by Kornecki et al. [19]. Then, Prandtl's lifting-line theory and the vortex lattice method were utilized to account for flags of finite aspect ratio. Good agreement with measurements was found for flags of large aspect ratio $(H / L>2)$. They attributed the disagreement between measurements and theory for inverted flags of small aspect ratio to the presence of a vortex-lift mechanism experienced by such flags. In the second study, Sader et al. [26] investigated the stability of inverted flags and rods with zero aspect ratio in a steady uniform flow. Excellent agreement was reported for flags of very small aspect ratios $(H / L<0.1)$. A Padé approximant was then employed to correlate the two solutions for zero and large aspect ratios, yielding a formula applicable over the entire range of aspect ratios studied.

On the computational fluid dynamics front, a two-dimensional numerical simulation on an inverted flag was conducted by Gurugubelli and Jaiman [16], who employed a higher-order fluid-structure interaction solver. They concluded that the inverted flag first loses stability by divergence and then tends to flutter due to a flow separation at the leading edge. Ryu et al. [25] contributed to the investigations on inverted flags by simulating the motions of a beam in reverse flow employing a two-dimensional immersed boundary method. In another study, Shoele and Mittal [30] investigated the energy harvesting performance of an inverted piezoelectric flag using the immersed boundary method. They examined the effect of initial inclination of the inverted flag to the impinging flow. The effect of aspect ratio was investigated by Tang et al. [32] via a three-dimensional simulation focusing on low Reynolds number flows. They found that the wider flags become unstable at lower critical flow velocities. 
The present paper explores the static instability and deformations of a low-aspect-ratio elastic inverted flag immersed in axial steady flow. It focuses on the time-invariant form of the fluid forces experienced by the flag (steady-flow model) to find the static solution of the governing equation. It is of interest on its own - as slender inverted flags lose stability via divergence - but it also serves to illustrate the modelling framework and solution techniques used in this study. In addition, this choice makes possible the investigation of the interplay between linear and nonlinear flow forces in the static response, in a less complicated manner. To this end, a theoretical model for low-aspect-ratio inverted flags is presented. A geometrically exact beam theory is coupled with a nonlinear aerodynamic theory. An integro-differential equation governing the motion of the inverted flag is derived within a Hamiltonian framework. The Galerkin scheme along with an adequate number of modes is utilised to obtain a compact system of nonlinear algebraic equations. The static response of the system is obtained using a Newton method. A thorough stability analysis is performed on the branches of the solution, making use of local geometric theory. Bifurcation diagrams with increasing flow velocity are systematically constructed. The effect of the aspect ratio as well as the amplitude of the initial curvature are investigated through performing extensive numerical simulations.

\section{Model development}

In this section, the dimensionless equations governing the motion of the system along with appropriate boundary conditions are developed within a Hamiltonian framework. The potential and kinetic energies of the inverted flag are formulated. Then, the fluid-related forces acting on the inverted flag are formulated based on a nonlinear steady theory. Finally, a model for initially curved inverted flags is presented.

\subsection{Kinetic and potential energies}

Figure 1 shows the system under consideration, which is a vertical cantilevered beam of length $L$, width $H$, thickness $h$, cross-sectional area $A$, surface area $S$, transverse moment of inertia $I$, and flexural rigidity $D$ immersed in an inviscid flow. The clamped end is located at the downstream region of the flow, while the flow impinges on the free end. The effect of gravity is neglected. The mechanical properties of the beam, i.e., its density, Poisson ratio, and Young's modulus are denoted by $\rho_{\mathrm{p}}, \nu$, and $E$, respectively; $\rho_{\mathrm{f}}$ stands for the density of the fluid flowing with mean flow velocity $U$. In the present paper, Euler-Bernoulli beam theory is employed to develop a nonlinear model for inverted cantilevered flags in axial flow; the rationale is outlined here: measurements by Kim et al. [18] show that the modeshapes of the flapping inverted flag can be

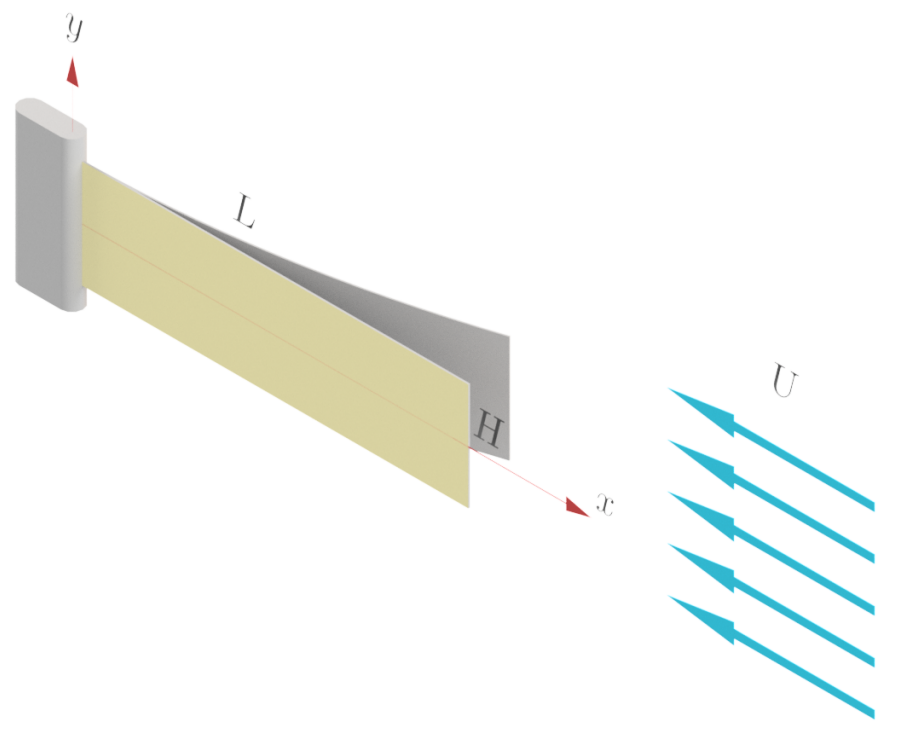

Figure 1: An inverted flag in axial flow; free at the upstream and clamped at the downstream end. 
decomposed using orthogonal beam modes. Accordingly, the transverse displacement of the flag, i.e. $w(x, t)$, is only a function of the axial coordinate and time, i.e. $x$ and $t$, respectively, and is symmetric with respect to the $y$ coordinate, as depicted in Fig. 1. Hence, the spanwise deformations are neglected due to symmetry. Furthermore, it was reported in experimental investigations [17, 31, 7, 41] and theoretical studies [11, 19, $34,15]$ on the flag-type configuration that the one-dimensional assumption for the displacement of the flag is reasonable. In fact, the spanwise deformation is negligible, at least at the onset of instability and for a considerable range of flow velocities beyond the critical point. Hence, the inverted flag undergoes two motions: $w(x, t)$, in transverse direction and $u(x, t)$, in the longitudinal direction. As such, the displacement field of a generic point on the cross-section of the flag at distance $z$ from the mid-line can be formulated as

$$
\mathbf{u}(x, z, t)=\left[\begin{array}{c}
u(x, t)-z \sin \psi(x, t) \\
0 \\
w(x, t)-z(1-\cos \psi(x, t))
\end{array}\right],
$$

where $\psi(x, t)$ denotes the rotation angle of the mid-line with respect to the $x$-axis (the slope of the mid-line). The relations

$$
\sin \psi(x, t)=\frac{\partial_{x} w(x, t)}{1+e(x, t)}, \quad \cos \psi(x, t)=\frac{1+\partial_{x} u(x, t)}{1+e(x, t)}, \quad \text { and } \quad \kappa(x, t)=\frac{\partial_{x} \psi(x, t)}{1+e(x, t)}
$$

hold, where $\kappa(x, t)$ and $e(x, t)$ are the curvature and the strain of the mid-plane, respectively. Note that $\partial_{t}$ and $\partial_{x}$ denote the derivatives with respect to time and the axial coordinate, respectively. The beam mid-line is assumed to be inextensible, i.e. $e(x, t)=0$, which yields: $\left(1+\partial_{x} u(x, t)\right)^{2}+\left(\partial_{x} w(x, t)\right)^{2}=1$. This equation necessitates that the transverse and longitudinal motions are not independent quantities. Therefore, among the three types of motion of the element (i.e. the transverse motion, $w(x, t)$, the longitudinal motion, $u(x, t)$, and the angle of rotation, $\psi(x, t))$, there is only one independent variable. Application of the inextensibility condition yields

$$
u(x, t)=\int_{0}^{x}(\cos \psi(s, t)-1) \mathrm{d} s \quad \text { and } \quad w(x, t)=\int_{0}^{x} \sin \psi(s, t) \mathrm{d} s .
$$

In this study, the longitudinal and transverse displacements are expressed as functions of the rotation angle. The model is thus constructed in terms of the angle of rotation, resulting in an integro-differential equation that governs the motion. The limitations regarding models expressed in terms of the transverse motion of the inverted flag are discussed in Appendix A.

The kinetic energy of the system involves the two non-vanishing components of the displacement field given in Eq. (1) and is obtained as follows:

$$
\begin{aligned}
T= & \frac{1}{2} \rho_{\mathrm{p}} \int_{0}^{L} \int_{A}\left(\left[\partial_{t}(u(x, t)-z \sin \psi(x, t))\right]^{2}+\left[\partial_{t}(w(x, t)-z(1-\cos \psi(x, t)))\right]^{2}\right) \mathrm{d} A \mathrm{~d} x \\
= & \frac{1}{2} \rho_{\mathrm{p}} A \int_{0}^{L}\left(\left[\int_{0}^{x} \sin \psi(s, t) \partial_{t} \psi(s, t) \mathrm{d} s\right]^{2}+\left[\int_{0}^{x} \cos \psi(s, t) \partial_{t} \psi(s, t) \mathrm{d} s\right]^{2}\right) \mathrm{d} x \\
& +\frac{1}{2} \rho_{\mathrm{p}} I \int_{0}^{L}\left(\left[\sin \psi(x, t) \partial_{t} \psi(x, t)\right]^{2}+\left[\cos \psi(x, t) \partial_{t} \psi(x, t)\right]^{2}\right) \mathrm{d} x .
\end{aligned}
$$

The potential strain energy of the inverted flag reads

$$
U=\frac{1}{2} E A \int_{0}^{L} e(x, t)^{2} \mathrm{~d} x+\frac{1}{2} D \int_{0}^{L} \kappa(x, t)^{2} \mathrm{~d} x=\frac{1}{2} D \int_{0}^{L}\left(\partial_{x} \psi(x, t)\right)^{2} \mathrm{~d} x,
$$

in which $D=E I /\left(1-v^{2}\right)$ is the plane-strain flexural rigidity for plates of large aspect ratios; in case of a slender inverted flag, the flexural rigidity becomes $D=E I$. The virtual work done by the fluid-related force 
normal to the flag is given by

$$
\delta W_{F}=\int_{0}^{L}\left(F_{\mathrm{N}}(x, t) \cos \psi(x, t) \delta w(x)-F_{\mathrm{N}}(x, t) \sin \psi(x, t) \delta u(x)\right) \mathrm{d} x,
$$

where $F_{\mathrm{N}}(x, t)$ represents the normal force acting on the flag, which is obtained in the next section. Substituting Eqs. (4)-(6) into the generalized Hamilton's principle and making use of the following equality [28]:

$$
\int_{0}^{L} g(x)\left(\int_{0}^{x} f(s) \delta \psi(s) \mathrm{d} s\right) \mathrm{d} x=\int_{0}^{L} f(x)\left(\int_{x}^{L} g(s) \mathrm{d} s\right) \delta \psi(x) \mathrm{d} x,
$$

one can obtain the following local equation for the transverse motion of the inverted flag in axial flow:

$$
\begin{aligned}
& \rho_{\mathrm{p}} I \partial_{t t} \psi-D \partial_{x x} \psi \\
& -\rho_{\mathrm{p}} A \sin \psi \partial_{t t}\left(\int_{x}^{L} \int_{0}^{s} \cos \psi(\eta, t) \mathrm{d} \eta \mathrm{d} s\right)+\rho_{\mathrm{p}} A \cos \psi \partial_{t t}\left(\int_{x}^{L} \int_{0}^{s} \sin \psi(\eta, t) \mathrm{d} \eta \mathrm{d} s\right) \\
& \quad+\cos \psi \int_{L}^{x} F_{\mathrm{N}}(s, t) \cos \psi(s, t) \mathrm{d} s+\sin \psi \int_{L}^{x} F_{\mathrm{N}}(s, t) \sin \psi(s, t) \mathrm{d} s=0,
\end{aligned}
$$

along with the associated clamped-free boundary conditions

$$
\psi(x=0, t)=0 \quad \text { and } \quad \partial_{x} \psi(x=L, t)=0 .
$$

\subsection{Fluid-related forces}

It has been shown experimentally that inverted flags of small aspect ratio do not display any flapping motion, but buckle abruptly instead [26]. In this case, it can be assumed that the flow reaches a steady-state and the flag loses its stability via a steady flow process. As such, steady and unsteady models are identical, and the system response is independent of time-dependent terms in the fluid forces [27]. For sufficiently large aspect ratios, on the other hand, the inverted flag displays a large-amplitude flapping motion. For these cases, the flow unsteadiness cannot be neglected and a fully-coupled fluidelastic problem needs to be tackled in order to capture the flapping dynamics. Nevertheless, it is worth showing that the equilibria obtained through the proposed steady model still exist as static solutions to the fully-coupled fluid-flag system.

In this section, a steady flow model based on the work of Bollay [5] is employed to derive an approximate expression for the aerodynamic loads acting on a plate. This allows us to identify the existence of the new equilibrium and the amplitude of deflection. Bollay's steady flow model is associated with small aspect ratio rectangular wings at high angle of attack $\alpha$ which assumes a uniform distribution of bound vortices along the chord of the plate. The flow separation around the side edges trails off at some angle $\vartheta$ to the free stream. Bollay obtained the induced velocity originating from the system of vortices at a generic point of the plate and calculated the normal force per unit length acting on the plate with the aid of the Kutta-Joukowski theorem [4]. Hence, the normal force per unit length can be expressed as

$$
F_{\mathrm{N}}=\frac{1}{2} \rho_{\mathrm{f}} H U^{2} C_{\mathrm{N}}
$$

where $C_{\mathrm{N}}$ is the normal force coefficient defined as

$$
C_{\mathrm{N}}=\pi \gamma(\vartheta)\left(\cos \alpha \sin \alpha+\sin ^{2} \alpha \tan \vartheta(1-g(R) \gamma(\vartheta))\right),
$$

and

$$
\tan \vartheta=\frac{\sin \alpha-\frac{1}{2} \gamma(\vartheta)\left(g(2 R)+\cot \vartheta\left(f_{1}(\zeta / 2)+f_{2}(\xi / 2)-\vartheta f_{3}(\lambda / 2)\right)\right)}{\cos \alpha+\frac{1}{2} \gamma(\vartheta)\left(\pi / 4+\left(f_{1}(\zeta / 2)+f_{2}(\xi / 2)-\vartheta f_{3}(\lambda / 2)\right)\right)}
$$


where $A=H / L$ represents the aspect ratio. The expressions of functions $\gamma(\vartheta, A), g(A), f_{1}(\zeta), f_{2}(\xi)$, $f_{3}(\lambda), \zeta(\vartheta), \xi(\vartheta)$, and $\lambda(\vartheta)$ are given in Appendix B. Bollay also determined the normal force mathematically for two asymptotic cases, for zero aspect ratio (i.e. $R=0$ ) and infinite aspect ratio (i.e. $R=\infty$ ), as

$$
\begin{array}{ll}
C_{\mathrm{N}}=2 \pi \sin \alpha \cos \alpha & \text { for } A=\infty, \\
C_{\mathrm{N}}=2 \sin ^{2} \alpha & \text { for } R=0 .
\end{array}
$$

The angle of the trailing vortices, $\vartheta(\alpha)$, can only be found by solving the nonlinear expression given in Eq. (12), numerically. Therefore, the expression for the normal force given in Eqs. (10) and (11) is not explicit. Gersten [13] employed Bollay's flow model and assumed that the flow trails off the surface of the plate at an angle equal to half of the plate's angle of attack, that is $\vartheta(\alpha)=\alpha / 2$. The corresponding expression for the normal force takes on the explicit form

$$
C_{\mathrm{N}}=\pi \gamma(\alpha / 2)\left(\sin (\alpha)-\gamma(\alpha / 2) \sin ^{2}(\alpha) g(R) \tan (\alpha / 2)\right) \text {. }
$$

This expression overestimates the normal force, especially for small aspect ratios. For instance, for the limiting case $A=0$, the normal force is equal to $C_{\mathrm{N}}=4 \sin ^{2} \alpha /(1+\cos \alpha)$, which is considerably larger than the force obtained without any assumption, see Eq. (13b).

Another method for finding an explicit form of the normal force is to approximate it on the basis of the Polhamus leading edge suction analogy [24] which states that the normal force acting on a wing at high angle of attack $\alpha$, can be expressed in the form of $K_{\mathrm{p}} \sin \alpha \cos \alpha+K_{\mathrm{v}} \sin ^{2} \alpha$ when leading-edge separation is involved. The coefficients $K_{\mathrm{p}}$ and $K_{\mathrm{v}}$ can be determined via an appropriate lifting-surface theory. For a value of the plate aspect ratio, the angle of the trailing vortices is calculated via solving the nonlinear equation (12) for a range of angles of attack, and the normal force coefficient can then be evaluated by inserting the resultant into the Eq. (11). Analogously to Polhamus's work, the normal force coefficient given in Eq. (11) can be recast in form of

$$
C_{\mathrm{N}}=K_{\mathrm{p}}(R) \sin \alpha \cos \alpha+K_{\mathrm{v}}(R) \sin ^{2} \alpha,
$$

with $K_{\mathrm{p}}(R)$ and $K_{\mathrm{v}}(R)$ being functions of the aspect ratio only. The normal force coefficient acting on the plate versus the angle of attack for four different aspect ratios is illustrated in Fig. 2. The curves obtained based on the explicit expression given in Eq. (15) with appropriate values for $K_{\mathrm{p}}(R)$ and $K_{\mathrm{v}}(R)$ are in perfect agreement with the values obtained from solving the implicit expression given in Eq. (12). Moreover, the expression given in Eq. (14) overestimates the normal force acting on the plate for small aspect ratios; there is also a discrepancy between the results for wider plates at higher angles of attack.

Figure 3 shows the trend of change in the $K_{\mathrm{p}}(R)$ and $K_{\mathrm{v}}(R)$ coefficients over a wide range of aspect ratios. It is worth mentioning that as the aspect ratio increases to very large values, $K_{\mathrm{p}}$ converges to $2 \pi$, whereas $K_{\mathrm{v}}$ approaches zero. On the other hand, for the other limiting case, i.e. zero aspect ratio, we have $K_{\mathrm{p}}=0$ and $K_{\mathrm{v}}=2$. This is again consistent with the limiting cases examined mathematically by Bollay, and given in Eq. (13a). For intermediate aspect ratios, $0<R<2, K_{\mathrm{p}}$ and $K_{\mathrm{v}}$ can be extracted from Figure 3(b) and utilised in numerical simulations.

The reactive force associated with the change in the fluid momentum in the transverse direction at each cross-section $x$ along a slender plate can be formulated in the steady limit as ${ }^{1}[20,39,6]$ :

$$
C_{\mathrm{R}}=\frac{\pi}{8} \rho_{\mathrm{f}} H^{2} U^{2}\left(3 \cos ^{2} \psi-1\right) \partial_{x} \psi
$$

\footnotetext{
${ }^{1}$ A more comprehensive approach, in the steady limit, may be to combine reactive and vortex-lift effects by extending Bollay's nonlinear lift theory to a plate with an arbitrary curvature along the length. However, this is a very challenging task, if not impossible, due to difficulties arising from the nonlinear nature of the equations (see [21]).
} 

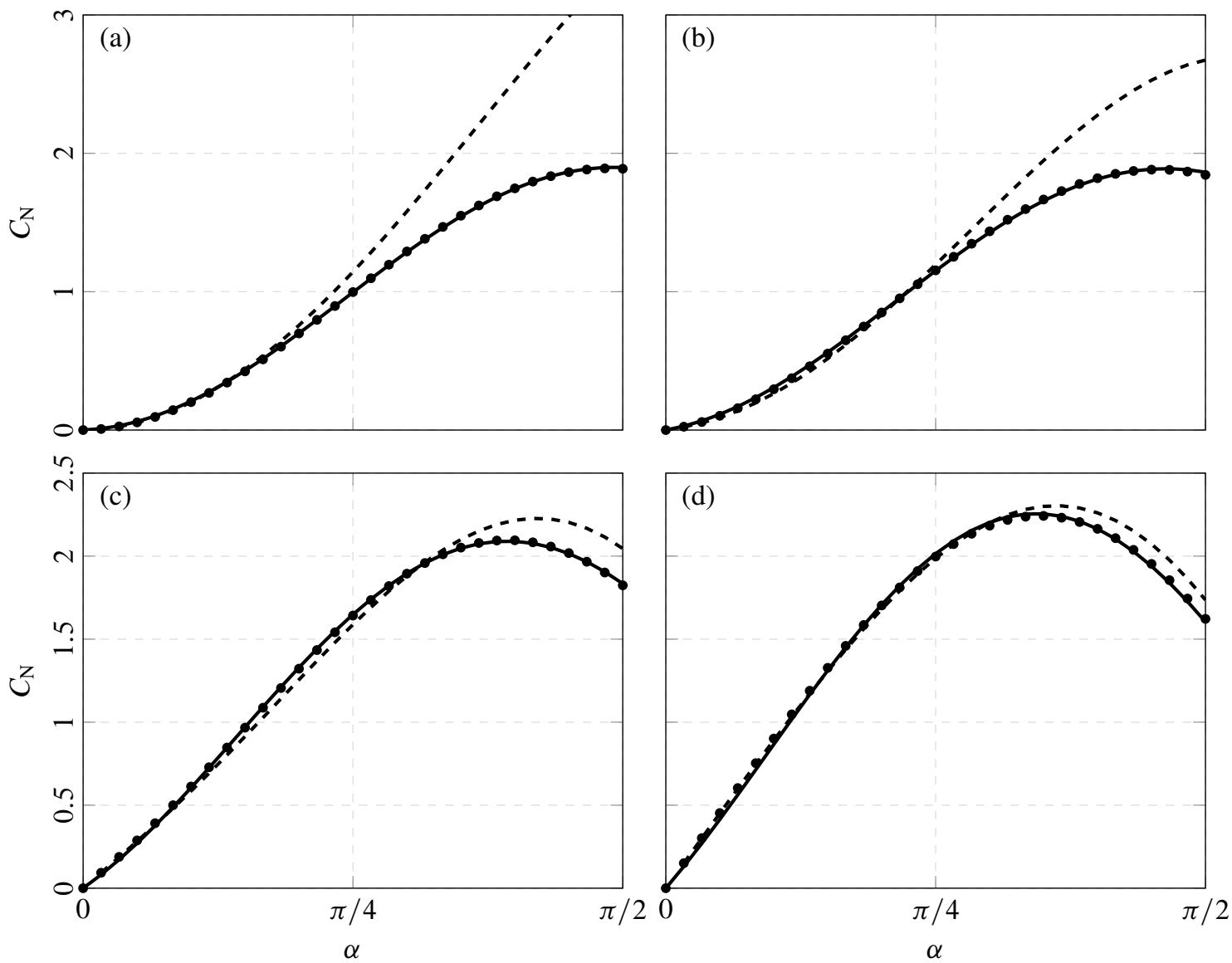

Figure 2: Normal force coefficient $C_{\mathrm{N}}$ versus the angle of attack $\alpha$. (a) $R=0.01$, (b) $R=0.1$, (c) $R=0.5$, and (d) $R=1.0 . C_{\mathrm{N}}$ calculated by solving Eq. (11) [॰], $C_{\mathrm{N}}$ approximated by the $\alpha / 2$-method [--.], and $C_{\mathrm{N}}$ obtained via Eq. (15) [-].
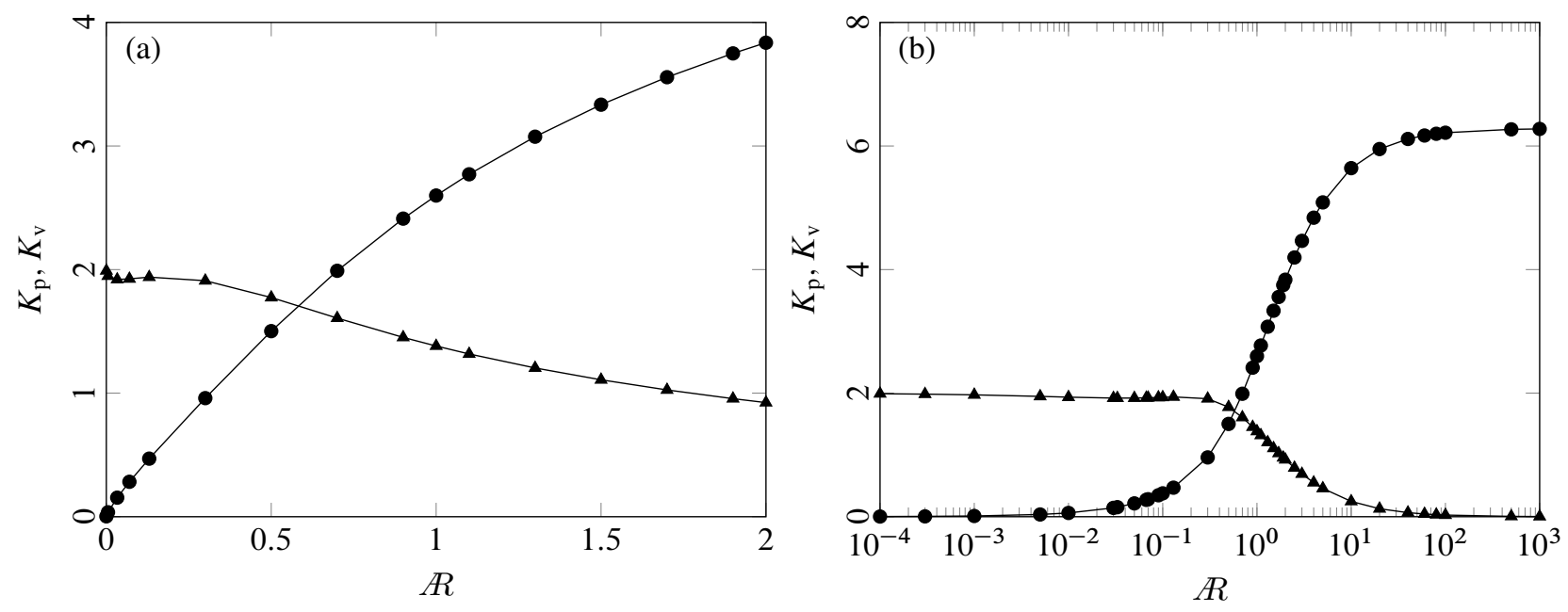

Figure 3: Variation of $K_{\mathrm{p}}[\bullet]$ and $K_{\mathrm{v}}[\mathbf{\Delta}]$ versus the aspect ratio $R$.

It should be noted that Bollay's model is predominantly associated with plates of small aspect ratios. The normal force coefficients obtained through this model have been compared with experiments and an excellent agreement was found for plates with very small aspect ratios (i.e. $R<0.66$ ) and good agreement was 
reported for $R=1.0$. For larger aspect ratios, however, the agreement was less satisfactory since some of the basic assumptions in the model are not fully satisfied (e.g. the assumptions of constant vortex strength and induced velocity across the span break down). Moreover, this model does not account for the contribution of the separated flow generated at the leading edge by the deformed plate at large amplitudes. Measurements show that the steady model proposed by Bollay can predict the normal force coefficient of a stationary thin low-aspect-ratio plate for angles of attack $\lesssim 40^{\circ}$ [5]. Hence, the model only provides an approximation of the fluid forces and can be utilized to identify the existence of the new equilibrium.

\subsection{Equation of motion}

Substituting the expressions in Eqs. (15) and (16) into Eq. (6) yields the virtual work done by the fluid-related force normal to the flag. Implementing this expression together with expressions for kinetic and potential energies given in Eqs. (4) and (5) in Hamilton's principle, performing the integration by parts, in addition to setting the coefficients of the variation of $\psi$ equal to zero, yields the following nonlinear integro-differential equation for the motion of the inverted flag subjected to axial flow:

$$
\begin{aligned}
& \rho_{\mathrm{p}} I \partial_{t t} \psi-\rho_{\mathrm{p}} A \partial_{t t}\left(\int_{x}^{L} \int_{0}^{s} \cos \psi(\eta, t) \mathrm{d} \eta \mathrm{d} s\right) \sin \psi+\rho_{\mathrm{p}} A \partial_{t t}\left(\int_{x}^{L} \int_{0}^{s} \sin \psi(\eta, t) \mathrm{d} \eta \mathrm{d} s\right) \cos \psi-D \partial_{x x} \psi \\
& +\frac{1}{2} \rho_{\mathrm{f}} H U^{2}\left[\operatorname { c o s } \psi \int _ { L } ^ { x } \left(\frac{\pi}{4} H\left(1-3 \cos ^{2} \psi(s, t)\right) \cos \psi(s, t) \partial_{s} \psi(s, t)+K_{\mathrm{p}}(R) \sin \psi(s, t) \cos ^{2} \psi(s, t)\right.\right. \\
& \left.+K_{\mathrm{v}}(R) \sin ^{2} \psi(s, t) \cos \psi(s, t)\right) \mathrm{d} s+\sin \psi \int_{L}^{x}\left(\frac{\pi}{4} H\left(1-3 \cos ^{2} \psi(s, t)\right) \sin \psi(s, t) \partial_{s} \psi(s, t)\right. \\
& \left.\left.\quad+K_{\mathrm{p}}(R) \sin ^{2} \psi(s, t) \cos \psi(s, t)+K_{\mathrm{v}}(R) \sin ^{3} \psi(s, t)\right) \mathrm{d} s\right]=0,
\end{aligned}
$$

along with the clamped-free boundary conditions given in Eq. (9). For ease of computation, the following dimensionless quantities are defined and utilised in numerical simulations:

$$
x^{*}=\frac{x}{L}, \quad t^{*}=\frac{t}{\tau}, \quad \Pi=\frac{\rho_{\mathrm{f}} S L^{2}}{D} U^{2}, \quad \beta=\frac{I}{A L^{2}}, \quad k_{\mathrm{p}}=\frac{K_{\mathrm{p}}}{2}, \quad k_{\mathrm{v}}=\frac{K_{\mathrm{v}}}{2},
$$

in which $\tau=\sqrt{\rho_{\mathrm{p}} A / D} L^{2}$. Note that the angle of attack in Eq. (15) is replaced by the slope of the flag mid-line, $\psi(x, t)$. Substituting these dimensionless parameters into Eq. (17) and dropping the asterisk notation in the interests of simplicity gives the dimensionless equation of motion as

$$
\begin{aligned}
\beta \partial_{t t} \psi & -\partial_{t t}\left(\int_{x}^{1} \int_{0}^{s} \cos \psi(\eta, t) \mathrm{d} \eta \mathrm{d} s\right) \sin \psi+\partial_{t t}\left(\int_{x}^{1} \int_{0}^{s} \sin \psi(\eta, t) \mathrm{d} \eta \mathrm{d} s\right) \cos \psi-\partial_{x x} \psi \\
+ & \Pi \times\left[\operatorname { c o s } \psi \int _ { 1 } ^ { x } \left(-\frac{\pi}{8} R\left(3 \cos ^{2} \psi(s, t)-1\right) \cos \psi(s, t) \partial_{s} \psi(s, t)\right.\right. \\
+ & \left.\frac{1}{2} \sin 2 \psi(s, t)\left(k_{\mathrm{p}}(R) \cos \psi(s, t)+k_{\mathrm{v}}(R) \sin \psi(s, t)\right)\right) \mathrm{d} s \\
+ & \sin \psi \int_{1}^{x}\left(-\frac{\pi}{8} R\left(3 \cos ^{2} \psi(s, t)-1\right) \sin \psi(s, t) \partial_{s} \psi(s, t)\right. \\
& \left.\left.+k_{\mathrm{p}}(R) \sin ^{2} \psi(s, t) \cos \psi(s, t)+k_{\mathrm{v}}(R) \sin ^{2} \psi(s, t) \sin \psi(s, t)\right) \mathrm{d} s\right]=0 .
\end{aligned}
$$

\subsection{Initial curvature}

A small initial tip deflection, $\Delta$, in the rage of $0.02<\Delta / L<0.04$ was reported in the experiment conducted by Kim et al. [18], originating from fabrication uncertainties: the flags were not perfectly flat. Moreover, Sader et al. [26] observed a slight initial deformation along the length of the flag, giving rise to a small initial break in 
symmetry of the system. In fact, they reported that the theoretical predictions systematically overestimate the measured values of critical flow velocity. This was presumably due to the presence of initial deformations along the flag length. Therefore, an initial curvature associated with zero initial stress, denoted $\psi_{0}(x)$, is considered in our model. Inserting this variable in the displacement field given in Eq. (1) yields

$$
\mathbf{u}(x, z, t)=\left[\begin{array}{c}
u(x, t)-z\left[\sin \left(\psi(x, t)+\psi_{0}(x)\right)-\sin \psi_{0}(x)\right] \\
0 \\
w(x, t)+z\left[\cos \left(\psi(x, t)+\psi_{0}(x)\right)-\cos \psi_{0}(x)\right]
\end{array}\right] .
$$

Deriving the expressions for the kinetic and potential energies as well as the virtual work done by the fluidrelated force via Hamilton's principle, and ignoring terms depending only on $\psi_{0}(x)$ (due to the zero initial stress condition), one obtains, with $\alpha(x, t)=\psi(x, t)+\psi_{0}(x)$

$$
\begin{aligned}
& \beta \partial_{t t} \psi-\sin \alpha \partial_{t t}\left(\int_{x}^{1} \int_{0}^{s} \cos (\alpha(\eta, t)) \mathrm{d} \eta \mathrm{d} s\right)+\cos \alpha \partial_{t t}\left(\int_{x}^{1} \int_{0}^{s} \sin (\alpha(\eta, t)) \mathrm{d} \eta \mathrm{d} s\right) \\
& -\partial_{x x} \psi+\Pi\left[\cos \alpha \int_{1}^{x} c_{\mathrm{N}}(s, t) \cos (\alpha(s, t)) \mathrm{d} s+\sin \alpha \int_{1}^{x} c_{\mathrm{N}}(s, t) \sin (\alpha(s, t)) \mathrm{d} s\right]=0
\end{aligned}
$$

where $8 c_{\mathrm{N}}(x, t)=\pi A\left(1-3 \cos ^{2} \alpha\right) \partial_{x} \alpha(x, t)+k_{\mathrm{p}} \sin \alpha \cos \alpha+k_{\mathrm{v}} \sin ^{2} \alpha$. Note that the angle of attack is replaced by $\alpha$ in order to account for the contribution of initial curvature in the aerodynamic loads. In this paper, the initial curvature is considered to be in the form of $\psi_{0}(x)=\Lambda \phi_{1}(x)$, with $\Lambda$ being the amplitude of the initial curvature and $\phi_{1}(x)$ denoting the first mode shape of a cantilevered beam. This results in a continuous, monotonically increasing deflection along the length of the flag, with the maximum amplitude at the tip denoted by $\Delta$.

\subsection{Discretized model}

In order to obtain a reduced-order model of the system, the following series expansion for the rotation angle $\psi$ can be considered:

$$
\psi(x, t)=\sum_{r=1}^{M} \phi_{r}(x) q_{r}(t)
$$

where $q_{r}(t)$ are the unknown time-dependent generalized coordinates and $\phi_{r}(x)$ are the eigenmodes of a cantilevered beam in vacuo, satisfying the clamped-free geometric boundary conditions. The steady analysis is performed by ignoring the time-derivative terms in the equation of motion; hence, the unknown variables, $q_{r}(t)$, are replaced by $q_{r}$. Time-derivative terms, however, are essential in performing the stability analysis on obtained solutions. It should be remarked that all the sin and cos terms are kept intact in the discretization and numerical simulations (they are not replaced by any order of approximations) in order to ensure reliable results even at large rotation amplitudes.

\section{Nonlinear static response}

In this section, a nonlinear static analysis is performed to obtain the critical values of the flow velocity corresponding to static bifurcations (buckling) and to construct the post-buckling configuration of the inverted flag. The nonlinear integro-differential equation given in Eq. (19) along with the corresponding boundary conditions given in Eq. (9) can be solved via two different techniques. In the first, the solution for $\psi(x)$ is obtained using a shooting technique for the boundary value problem at hand. The second technique is to discretize the system with the aid of the Galerkin method, using appropriate mode shapes satisfying the boundary conditions and to reduce the equations to a set of nonlinear algebraic equations; the resultant set of equations is solved using a Newton method. The response of the system is obtained using both methods and the 
results are presented in Fig. 4; the bold line shows the feasible solutions for $\psi(x)$ using the first method and the dashed line illustrates those obtained using the second method involving four modes; each line corresponds to a configuration of the inverted flag (shown in subfigure 4(b)) and indicates the slope along the axial coordinate (i.e. $x$ ). As seen, the difference between the two solutions is negligible, demonstrating the reliability of the
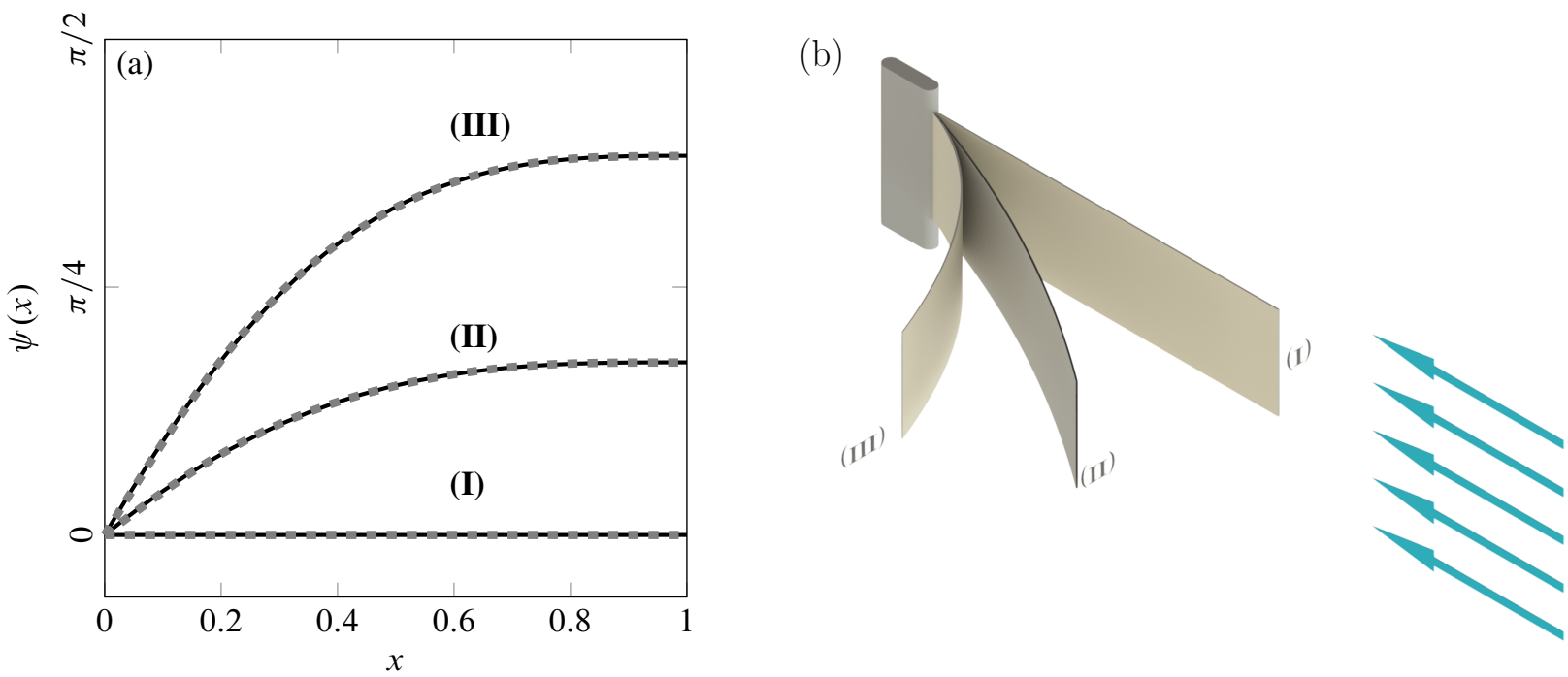

Figure 4: (a) Three branches of solution of a system with $R=0.25, \Delta / L=0.0$, and $\Pi=8.0$; solution obtained by shooting [-] and Galerkin [-- $]$ techniques, (b) configuration of the inverted flag associated with each branch of the solution.

second method and sufficiency of the number of modes utilized in the Galerkin scheme. Subfigure 4(a) shows three possible solutions for $\psi(x)$ at $\Pi=8$, representing three equilibrium configurations for the inverted flag. These solutions can be either stable or unstable; a stability analysis is conducted based on local geometric theory [1] and bifurcation points are identified mathematically. A pitchfork bifurcation materializes when one eigenvalue vanishes and becomes equal to zero; if the real-part of a pair of complex conjugate eigenvalues becomes positive (it crosses the imaginary-eigenvalue axis), a Hopf bifurcation takes place.

Figure 5 shows the bifurcation diagrams for an inverted flag with $R=0.1$; the flow velocity is varied as the bifurcation parameter. Bold and dashed lines represent stable and unstable branches of the solution, respectively. Subfigures 5(a) and 5(b) illustrate the slope at the free end, the tip transverse displacement (the maximum transverse amplitude of buckling), and tip longitudinal displacement, respectively. As seen in this figure, the inverted flag is initially stable for relatively small flow velocities and resides at its original equilibrium position. Two new solutions are found at point $\mathrm{A}$ where $\Pi_{\mathrm{P}}=33.8$; these branches are initially unstable and become stable as the flow velocity is reduced to the folding at point $\mathrm{B}$ via a saddle-node bifurcation where $\Pi_{\mathrm{S}}=8.7$. This characterizes a strong subcritical pitchfork bifurcation (divergence); there are three stable equilibrium solutions, along with two unstable ones in this range of flow velocity (i.e. $\Pi=[8.7,33.8]$ ). The basin of attraction of the trivial solution is represented by the unstable solution; it becomes smaller as $\Pi$ approaches $\Pi_{P}$. Hence, even at flow velocities much smaller than the critical value, the inverted flag may exhibit a jump from the trivial solution (original equilibrium configuration) to the stable bifurcated solution (deflected equilibrium configuration) due to small perturbations. More specifically, the unstable branch is not physically possible, meaning that if the flag is perturbed from its original equilibrium position to the unstable position, it gravitates towards deflected or undeflected stable configurations.

The perturbations may be imposed externally (e.g. an external pole) or may arise from fluctuations in the impinging flow. In the latter case, small perturbations about the static equilibrium die out, and the system returns to the origin; perturbations of amplitude larger than the amplitude of the unstable branch, on the other hand, lead the system away from the trivial equilibrium and into the deflected configuration at a flow velocity 

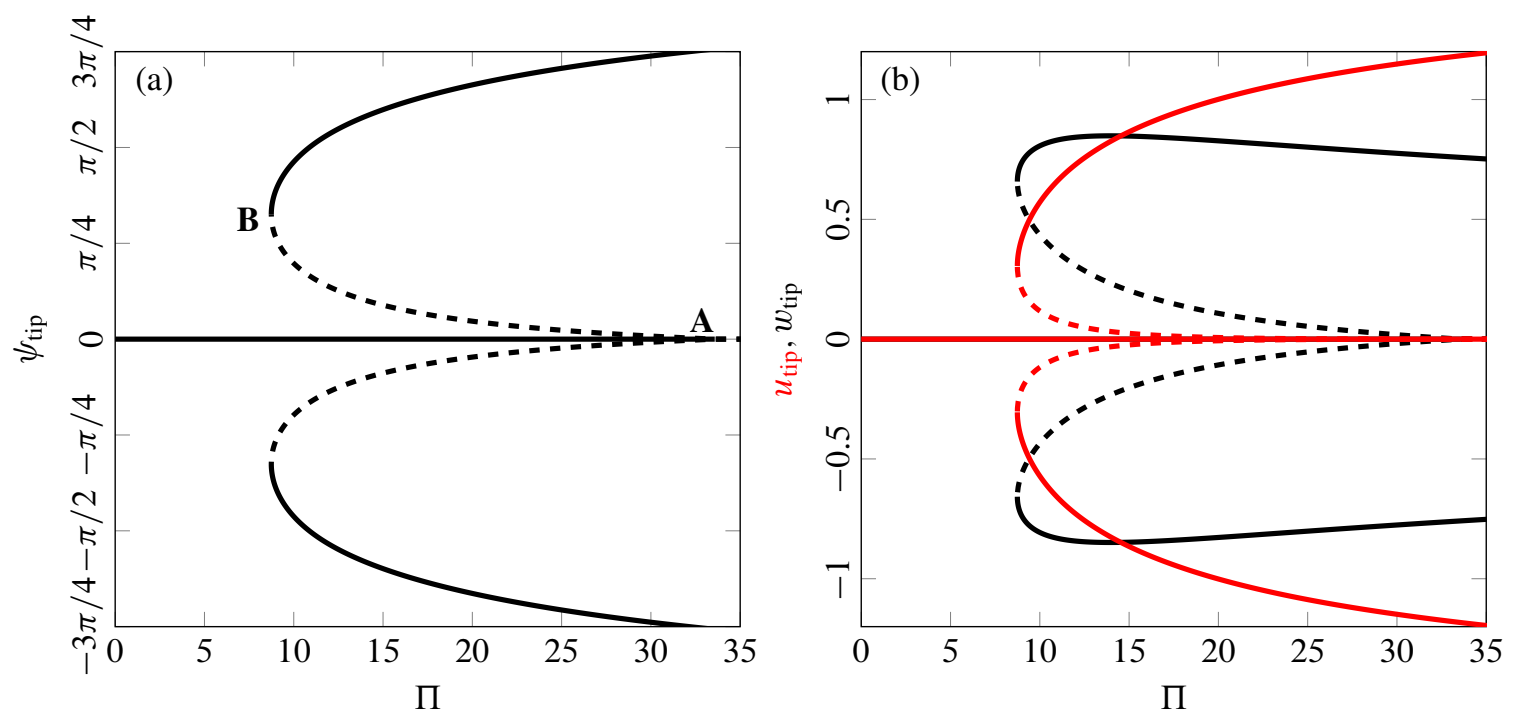

Figure 5: Bifurcation diagrams of a perfectly flat inverted flag with $R=0.1$. (a) tip slope of the flag, (b) tip transverse/longitudinal displacements. Pitchfork bifurcation at $\Pi_{P}=33.8$ and saddle-node bifurcation at $\Pi_{S}=8.7$; stable static solution (undeflected stable equilibria) [-], unstable static solution [-..].

less than $\Pi_{P}$.

It should be noted that the symmetrical response of the system with respect to the horizontal axis is the reason for buckling of the inverted flag to either side. The initial condition of the flag (such as small amplitude of an initial inclination) determines its final configuration (it may bend to one or the other of the deflected positions).

An initial curvature is taken into account in the modelling and simulations and the results are presented in Fig. 6; note that the initial curvature is introduced in the form of the first-mode shape of the cantilevered beam
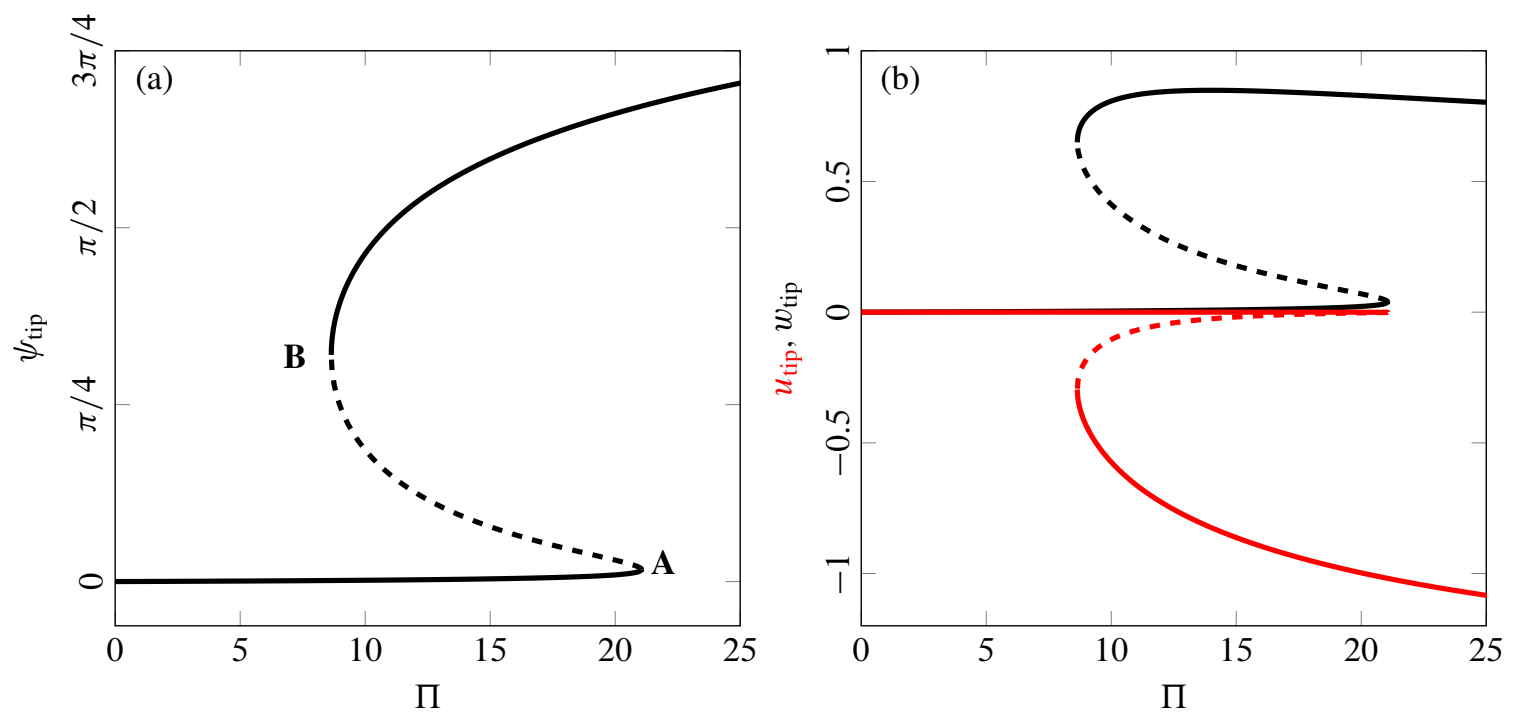

Figure 6: Bifurcation diagrams of an initially curved inverted flag with $R=0.1$ and initial tip deflection, $\Delta / L=0.01$. (a) tip slope of the flag, (b) tip transverse/longitudinal displacements. Two saddle-node bifurcations at $\Pi_{\mathrm{S} 1}=21.1$ and $\Pi_{\mathrm{S} 2}=8.6$; stable static solution (undeflected stable equilibria) [-], unstable static solution $[\cdot-\cdot]$. 
and a small amplitude of the initial curvature is considered. The amplitude of the initial curvature, $\Lambda$ is selected in a way that the inverted flag possesses a very small initial tip deflection equal to $\Delta / L=0.01$. As seen, the pitchfork bifurcation is destroyed due to the presence of the initial curvature and is replaced by a saddle-node bifurcation. Hence, the response of the inverted flag displays a continuous deformation path incorporating two saddle-node bifurcations; the equilibrium solution becomes unstable at the first saddle-node bifurcation (point A) and regains stability at the second one (point B). In particular, at sufficiently small flow velocity, the system remains at its original equilibrium position. Increasing the flow velocity gives rise to an abrupt jump at point $\mathrm{A}$ where $\Pi_{\mathrm{S} 1}=21.1$ from the trivial equilibrium position to a configuration of larger amplitude. A reverse scenario is expected when the inverted flag is at the buckled position and the flow velocity is decreased; i.e. the flag jumps back to the original configuration at point $\mathrm{B}$ where $\Pi_{\mathrm{S} 2}=8.6$. Furthermore, considering initial curvatures in the model gives rise to a significant reduction in the critical value of the flow velocity, which is now determined by the first saddle-node bifurcation point. The second saddle-node bifurcation, on the other hand, is only slightly shifted to lower flow velocities. Remarkably, as opposed to the prediction for a perfectly flat model, even the inverted flag of zero aspect ratio studied by Sader et al. [26] is expected to jump to the deflected equilibrium at a finite flow velocity; note that the perfect one remains stable and resides at its undeflected configuration for considerably large flow velocities in the absence of external perturbation.

The sensitivity of the model to initial curvature along the length of the inverted flag is examined next, and the results are plotted in Fig. 7. The amplitude of the initial curvature is selected in a range such that the

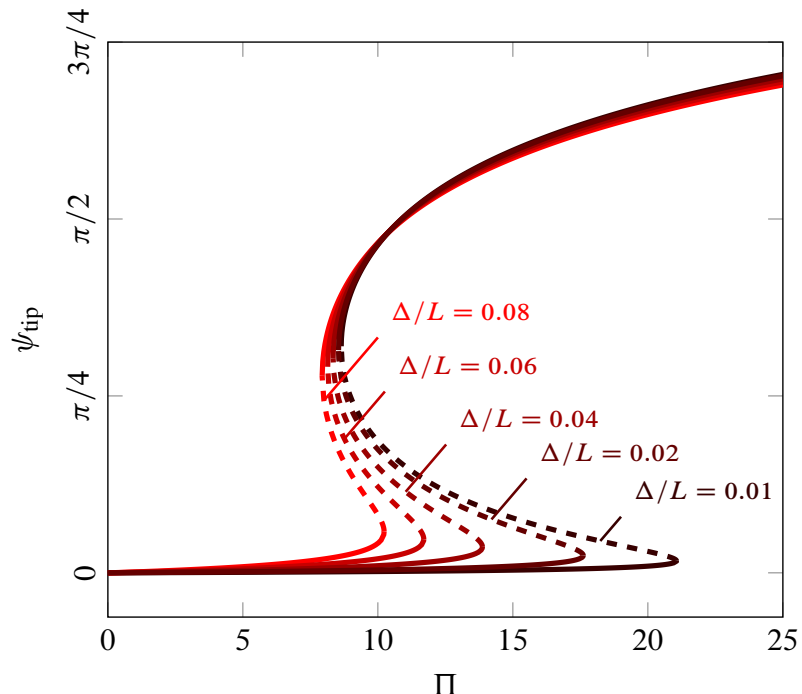

Figure 7: Bifurcation diagram of the inverted flag with $R=0.1$ for various initial tip deflections $\Delta / L$.

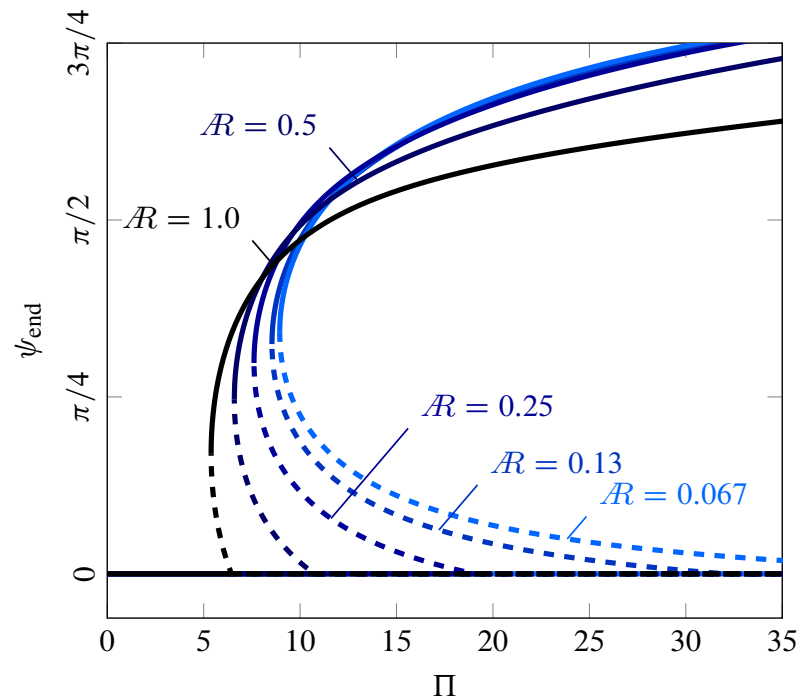

Figure 8: Bifurcation diagrams of the inverted flag for various aspect ratios $R$.

inverted flag exhibits tip deflections satisfying $0.01<\Delta / L<0.08$. The presence of an initial curvature leads to a modification in the stiffness matrix of the system, which in turn yields a different deflected equilibrium state. As shown, larger initial curvature amplitudes result in a significant change in the response of the system. More specifically, the first saddle-node bifurcation shifts to substantially smaller values of the flow velocity. In contrast, the second saddle-node bifurcation only shifts slightly. Additionally, the general behaviour of the inverted flag incorporating an initial curvature remains subcritical and the flag deformation jumps abruptly from a relatively small amplitude to a substantially larger one with a very small increment in the flow velocity.

Figure 8 displays the sensitivity of the static response of the inverted flag to its aspect ratio. First, the coefficients $K_{\mathrm{p}}$ and $K_{\mathrm{v}}$ of the fluid load on the plate are calculated for any aspect ratio. Then, the equation of motion is solved and the stability of the response is explored. As seen in the plot, the critical flow velocity decreases monotonically as the aspect ratio is increased. This is in good agreement with experimental observations and CFD model predictions in the literature [27, 32]. For instance, the present theory predicts 
$\Pi_{\mathrm{P}}=42.5$ and $\Pi_{\mathrm{S}}=8.9$ for a slender inverted flag with $A=0.067$, whereas it predicts $\Pi_{\mathrm{P}}=4.6$ and $\Pi_{\mathrm{S}}=5.2$ for a square one with $R=1$. This shows the significant effect of the finite aspect ratio on the nonlinear behaviour of the system. Furthermore, as reported by Sader et al. [26], the subcritical behaviour of the system becomes weaker for wider flags and the multiple equilibria merge; their measurements revealed that these multiple equilibria occur only for $R=1.7$. Strikingly, the bistable region obtained for a perfectly flat inverted flag of $R=1.7$ is insignificant. In fact, for this case, the velocity range of bistability (i.e. $\left[\Pi_{\mathrm{S}} \Pi_{\mathrm{P}}\right]$ ) is very narrow, resulting in $\left(\Pi_{P}-\Pi_{S}\right) / \Pi_{P} \simeq 8 \%$. More importantly, if a very small initial curvature with an initial tip deflection of $\Delta / L=0.004$ is considered in the model, the bistable zone disappears completely. Moreover, this figure highlights that the critical points shift to smaller flow velocities, meaning that a flags of larger aspect ratio is more vulnerable to instability. A comparison between the obtained critical values of the flow velocity via the proposed model (i.e., the pitchfork bifurcation, $\Pi_{\mathrm{P}}$, as well as the saddle-node bifurcation, $\Pi_{S}$ ) and those measured by Sader et al. [26] is given in Table 1. To extract the value of $\Pi_{P}$

\begin{tabular}{lccccccccccc}
\hline Aspect ratio of the flag $(R)$ & 0.067 & 0.1 & 0.13 & 0.5 & 1.0 & 0.067 & 0.1 & 0.13 & 0.5 & 1.0 \\
\hline & \multicolumn{8}{c}{ dimensionless flow velocity } & $\Pi_{\mathrm{P}}$ & \multicolumn{6}{c}{ dimensionless flow velocity $\Pi_{\mathrm{S}}$} \\
Experiment (Sader et al. [26]) & - & $\gtrsim 24$ & $13-26$ & $10-15$ & $6-10$ & 9.1 & 8.6 & 8.1 & 6.7 & 5.5 \\
Current model & 49.7 & 33.8 & 32.0 & 10.7 & 5.4 & 8.9 & 8.7 & 8.5 & 6.6 & 6.4 \\
\hline
\end{tabular}

Table 1: Critical flow velocities for the pitchfork and the saddle-node bifurcations: comparison between the current theory and measurements of Sader et al. [26].

from the experimental results given in [26], it is assumed that the inverted flag possesses a small initial tip deflection $0.02<\Delta / L<0.04$ due to imperfections. This leads to tip rotation angles of approximately $0.027<\psi_{0}(L)<0.055$. Unstable measured points in [26] with amplitudes larger than 0.027 or 0.055 are considered as weakly deflected unstable equilibria, while those with smaller amplitudes are considered as undeflected. As such, a range of dimensionless flow velocity for the experimental value of $\Pi_{P}$ is reported in Table 1 for every aspect ratio. The comparison shows an acceptable agreement between the current theoretical predictions and the reported experimental measurements. A better match is found for flow velocities at which the saddle-node bifurcations take place.

It should be emphasised that according to the experiments by Sader et al. [26], flags with $R=0.5$ and $R=1.0$ display large-amplitude flapping motions. As noted earlier, the use of the steady model removes the possibility of flapping dynamics. In these cases, large-amplitude flapping is the global attractor for the inverted-flag system, and the deformed and undeformed equilibria obtained through the proposed steady model are not stable. The presence of these unstable equilibria and the associated flag shapes were assessed by Sader et al. [26] by lightly touching the flapping flags with a thin rigid pole. Comparing the measurements with predictions of the proposed model for $R=0.5$ and $R=1.0$ shows that the steady-flow model works quite well, even for these aspect ratios, in predicting the critical flow velocities.

The system response for various aspect ratios in the presence of a slight initial curvature is examined and the results are plotted in Fig. 9: $\Lambda$ is selected such that the initial tip deflection is $\Delta / L=0.02$. As seen, the pitchfork bifurcation is replaced by a saddle-node bifurcation at relatively lower flow velocities. Increasing the aspect ratio results in a concurrent shift in both saddle-node bifurcations. The first one shifts to a higher tip deflection and the second one moves toward lower tip deflections. This results in a narrower bistable zone which weakens the subcritical behaviour of the system. In fact, at $R=1.0$, the bistable zone disappears completely and the response of the system becomes supercritical. In this case, the behaviour is not dependent on the initial conditions and the inverted flag buckles at $\Pi=4$.1. For larger aspect ratios, the abrupt jump phenomenon in the response of the system is replaced by a bending behaviour, and the change in the amplitude of the response becomes smoother.

It would be interesting to determine the aspect ratio at which the response of the initially curved system switches from a subcritical divergence to a supercritical one. In this study, this aspect ratio is referred to as the 


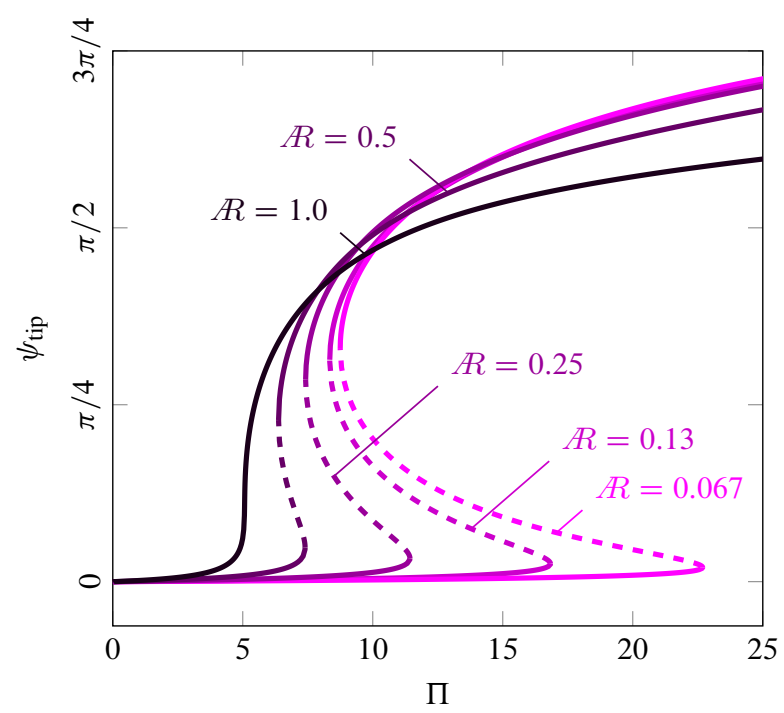

Figure 9: Bifurcation diagrams of the initially curved inverted flag for various $R$ and $\Delta / L=0.02$.

critical aspect ratio denoted $R_{\mathrm{cr}}$. Figure 10 shows the response of several inverted flags with different initial curvature magnitudes and the corresponding critical values of the aspect ratio. For instance, an inverted flag possessing an initial tip deflection equal to $\Delta / L=0.01$ exhibits subcritical behaviour if its aspect ratio is smaller than 1.15. The response is supercritical, on the other hand, for aspect ratios exceeding 1.15. Moreover, $R_{\text {cr }}$ decreases with larger initial curvatures. It is recalled that the stable deflected solutions shown for flags of sufficiently large aspect ratios (say, above 0.5 ) are not expected on physical grounds, since in these cases the inverted flag displays a large-amplitude flapping motion.

Similarly, the critical initial curvature referred to as $\Lambda_{\mathrm{cr}}$ is defined for a specific aspect ratio in which the system response changes from a subcritical behaviour to a supercritical one. The critical initial curvatures for different aspect ratios are obtained and the results are reported in Table 2. These results emphasize that, for small aspect ratios, a large initial curvature is required in order to weaken the subcritical behaviour of the inverted flag. For large aspect ratios, on the other hand, very small initial curvatures guarantee a supercritical response of the system.

\begin{tabular}{lcccccccc}
\hline Aspect ratio $(R)$ & 0.01 & 0.1 & 0.25 & 0.5 & 0.75 & 1.0 & 1.3 & 1.7 \\
Critical initial tip deflection $\Delta_{\text {cr }} / L$ & 0.25 & 0.17 & 0.12 & 0.055 & 0.028 & 0.015 & 0.010 & 0.004 \\
\hline
\end{tabular}

Table 2: Critical initial tip deflections $\Delta_{\mathrm{cr}} / L$ (at which the response of the system alters from the supercritical type to the subcritical type) for various aspect ratios $R$.

\section{Conclusions}

The present study investigated the nonlinear static response and deformations of low-aspect-ratio inverted flags subjected to a uniform steady axial flow. A theoretical model for low-aspect-ratio inverted flags was presented taking into account the effect of initial curvature along the length of the flag. Hamilton's principle was used to derive a one-dimensional nonlinear partial integro-differential equation of motion. The equation was discretized using the Galerkin method, and solved by means of a Newton method. Bifurcation diagrams were plotted selecting the flow velocity as the bifurcation parameter. A thorough stability analysis was conducted on the branches of the solution based on local geometric theory. The numerical results highlighted that: 

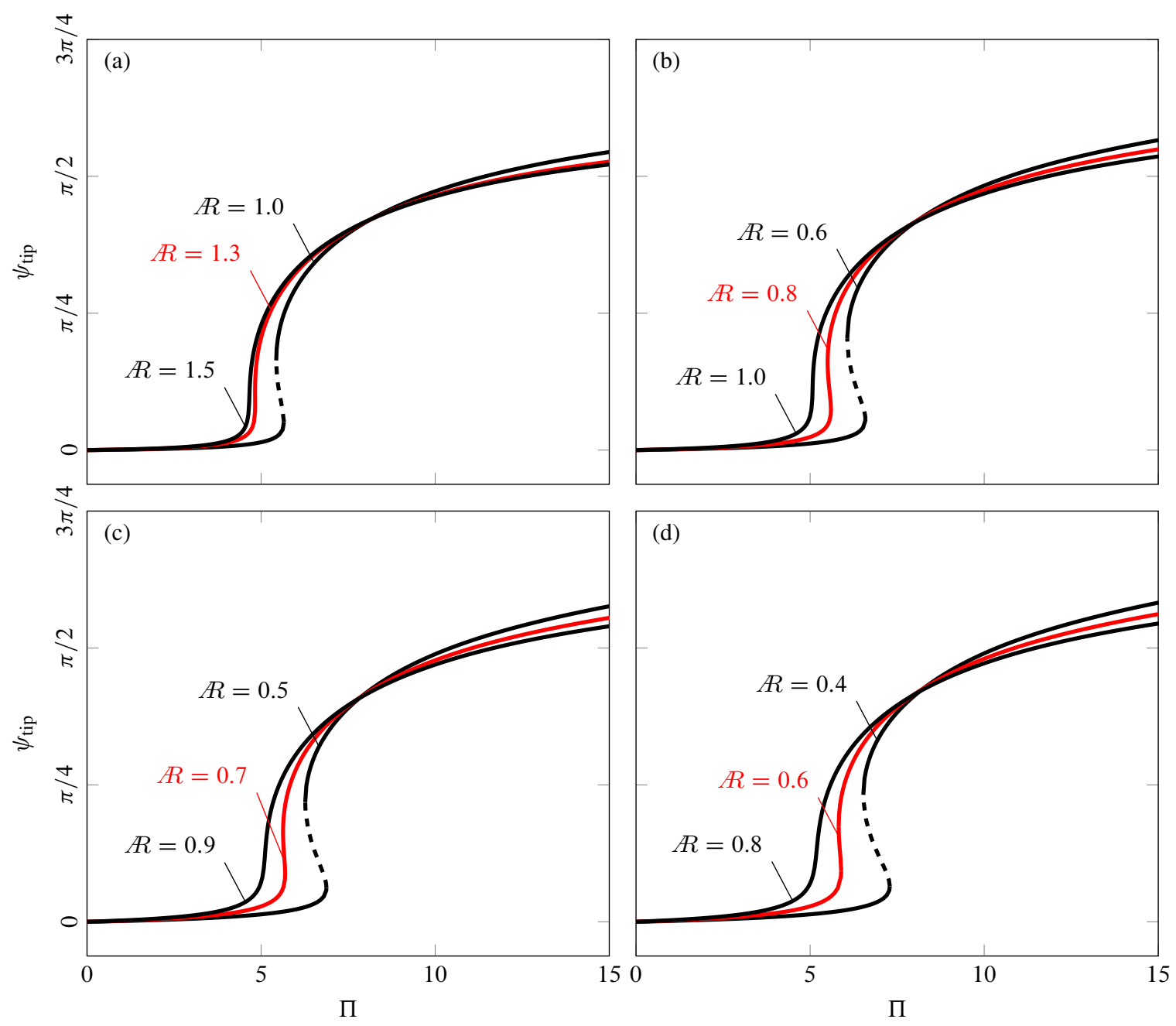

Figure 10: Bifurcation diagrams of the initially curved inverted flags for various initial tip deflections and $R_{\text {cr }}$ such that the system response alters from supercritical type to subcritical type. Inverted flag response with (a) an initially curved inverted flag with $\Delta / L=0.01$ corresponding to $R_{\mathrm{cr}}=1.3$, (b) an initially curved inverted flag with $\Delta / L=0.02$ corresponding to $R_{\text {cr }}=0.8$, (c) an initially curved inverted flag with $\Delta / L=0.03$ corresponding to $R_{\mathrm{cr}}=0.70$, and (d) an initially curved inverted flag with $\Delta / L=0.04$ corresponding to $R_{\text {cr }}=0.6$.

1. Typically, a perfectly flat inverted flag resides at its original static equilibrium at relatively low flow velocities and loses stability by static divergence via a subcritical or supercritical pitchfork bifurcation, depending on the flag aspect ratio. The non-trivial attracting solution (deflected position) of the transverse motion of inverted flags of sufficiently small aspect ratio is stable. In these cases, the unstable branch of the solution is bracketed by a pitchfork and a saddle-node bifurcation and determines a margin for coexisting attractors around the equilibria.

2. The larger the span, the less stable the system. The critical flow velocities associated with the pitchfork and saddle-node bifurcation points shift to lower magnitudes. Moreover, the subcritical behaviour of the system becomes weaker for wider flags and the existence of multiple equilibria vanishes for a specific aspect ratio; the response of the system switches to a subcritical pitchfork bifurcation.

3. Considering a small disturbance on the initial curvature of the flag replaces the pitchfork bifurcation by a primary saddle-node bifurcation, at lower critical flow velocities. The response of the initially curved system exhibits two saddle-node bifurcations associated with two jumps from the undeflected 
equilibrium to the deflected one.

4. Increasing the initial curvature amplitude alters the position of the first saddle-node bifurcation dramatically. The second saddle-node bifurcation, on the other hand, shifts only slightly to lower flow velocities. For sufficiently large values of initial curvature amplitude, the bistable zone in the response of the system becomes narrower up to the point that the response of the system switches from a subcritical type to supercritical one.

5. It is remarked that the steady potential flow model employed in this study only gives a rough approximation of the exact fluid forces for large angles of attack where viscous effects become dominant, and in turn gives only an approximation of the flag distorted shape. However, the good agreement between the results obtained via the present model and measurements in the literature signifies that the steady form of the fluid force governs the onset of instability and buckling of inverted flags of small aspect ratios.

Acknowledgment. The financial support by the Solution Mining Research Institute (SMRI), Pipeline Research Council International (PRCI) and the Natural Sciences and Engineering Research Council of Canada (NSERC) is gratefully acknowledged.

\section{Appendix A. Comparison between models corrected up to different orders}

The nonlinear equations governing the transverse motion of a cantilevered beam or plate can be obtained either by Newtonian or Hamiltonian methods. Routinely, the equations are derived in terms of the transverse displacement and are reliable up to a certain point in the derivation. In fact, in the derivation procedure, the curvature is expressed as a function of the transverse displacement, making the implicit assumption that the angle of the rotation is limited to the range of $[-\pi / 2, \pi / 2]$. This assumption is reliable in many studies where the deformation amplitude is not very large. In most of the studies, the equations of motion are truncated up to cubic order in terms of the transverse displacement, $w(x, t)$. Supposing relatively large deformations, a larger order of magnitude should be selected in order to obtain more reliable results. However, if the system at hand exhibits very large deformations, even higher-order models fail to predict the response of the system correctly.

In order to show the limitation of the model derived in terms of the transverse displacement, the steady form of the equation of motion for the inverted flag given in Eq. (19) is recast based on the transverse displacement of the beam:

$$
\begin{aligned}
& \partial_{t t} w-\partial_{x}\left[\frac{\partial_{x} w}{\sqrt{1-\left(\partial_{x} w\right)^{2}}} \int_{1}^{x} \int_{0}^{s} \partial_{t t} \sqrt{1-\left(\partial_{\xi} w(\xi, t)\right)^{2}} \mathrm{~d} \xi \mathrm{d} s+\partial_{x}\left(\frac{\partial_{x x} w}{\sqrt{1-\left(\partial_{x} w\right)^{2}}}\right) \frac{1}{\sqrt{1-\left(\partial_{x} w\right)^{2}}}\right] \\
& -\frac{\Pi}{\sqrt{1-\left(\partial_{x} w\right)^{2}}}\left[k_{\mathrm{v}}\left(\partial_{x} w\right)^{2}+k_{\mathrm{p}} \partial_{x} w \sqrt{1-\left(\partial_{x} w\right)^{2}}+\frac{\pi R}{8}\left(\partial_{x} w \partial_{x} \sqrt{1-\left(\partial_{x} w\right)^{2}}-2 \partial_{x}\left(\partial_{x} w \sqrt{1-\left(\partial_{x} w\right)^{2}}\right)\right)\right] \\
& +\frac{\Pi \partial_{x x} w}{\left(1-\left(\partial_{x} w\right)^{2}\right)^{\frac{3}{2}}} \int_{x}^{1}\left[k_{\mathrm{v}}\left(\partial_{s} w(s, t)\right)^{2}+k_{\mathrm{p}} \partial_{s} w(s, t) \sqrt{1-\left(\partial_{s} w(s, t)\right)^{2}}\right. \\
& \left.+\frac{\pi R}{8}\left(\partial_{s} w(s, t) \partial_{s} \sqrt{1-\left(\partial_{s} w(s, t)\right)^{2}}-2 \partial_{s}\left(\partial_{s} w(s, t) \sqrt{1-\left(\partial_{s} w(s, t)\right)^{2}}\right)\right)\right] \partial_{s} w(s, t) \mathrm{d} s=0 .
\end{aligned}
$$

Note that the inertia terms are retained. Thereupon, three truncated models of the equation at hand are considered, namely the third order, $O\left(\epsilon^{3}\right)$, the fifth order, $O\left(\epsilon^{5}\right)$, the seventh order, $O\left(\epsilon^{7}\right)$, of magnitude, where $O(\epsilon)$ denotes the first order. Additionally, one model without any order of approximation (i.e., exact with respect to $w(x, t))$ is taken into account. The resultant equations are discretized with the aid of Galerkin's technique and solved numerically.

Figure A.1 shows the bifurcation diagram of an inverted flag of $R=0.1$ and $\Delta / L=0.01$ for the various approximations mentioned above. The "exact" curve is associated with the case in which the terms under the square-root are not replaced by truncated Taylor series. As seen in this figure, the third-order approximation, which is widely used in the literature, overestimates the response of the system. At a certain flow velocity, the 


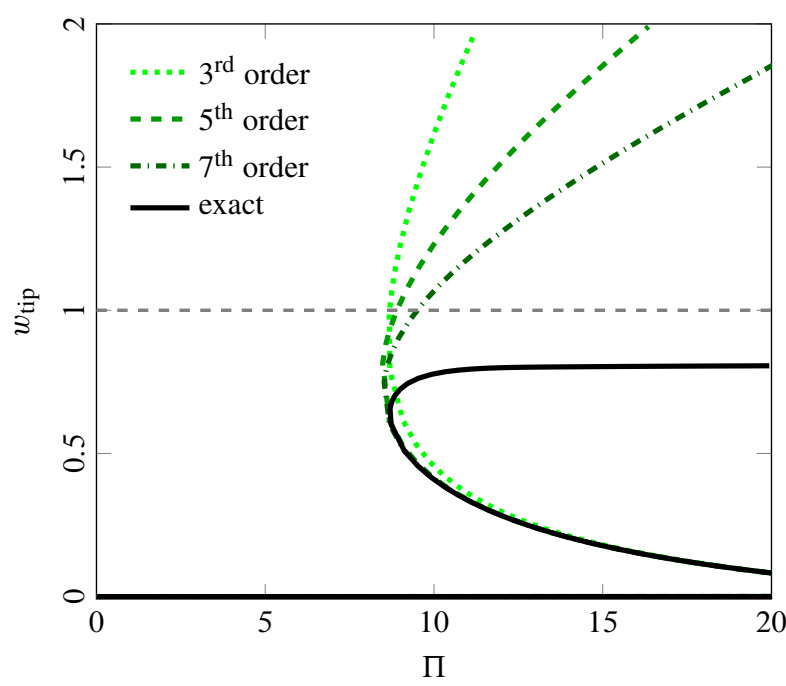

Figure A.1: Bifurcation diagrams for an inverted flag with $R=0.1$ and $\Delta / L=0.01$, using different truncated models of Eq. (A.1).

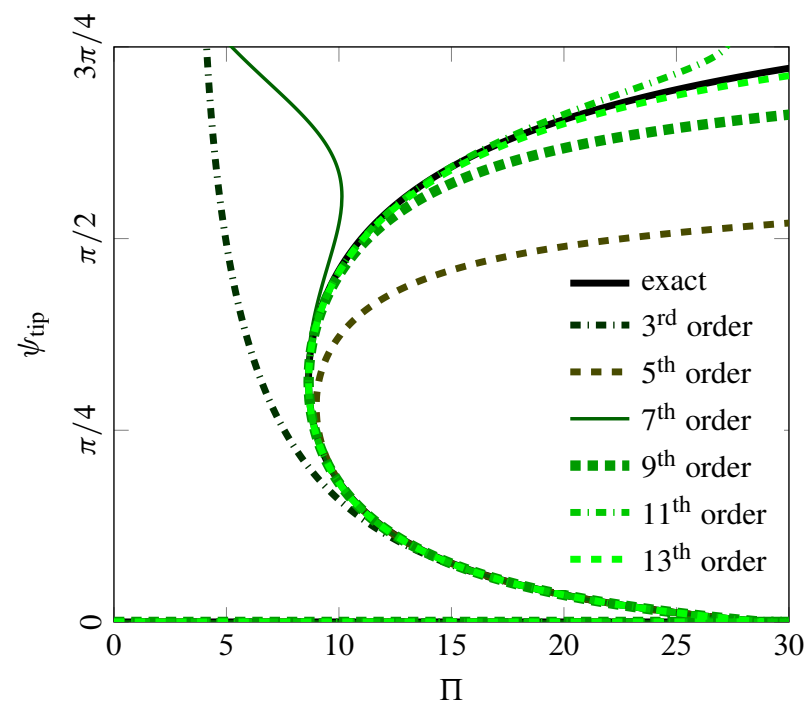

Figure A.2: Bifurcation diagrams for an inverted flag with $R=0.1$ and $\Delta / L=0.01$, using different truncated models of Eq. (19).

amplitude of the deflection is even larger than the length of the inverted flag! Higher-order approximations (i.e. the fifth and the seventh order) are more reliable. However, they are not capable of predicting the behaviour of the system at high flow velocities where the inverted flag undergoes very large deformations. It is interesting to see that even the exact solution cannot predict the response of the system correctly. In fact, the amplitude of the transverse tip deflection converges to 0.79 . This signifies that there is a flow velocity at which the tip angle exceeds $\pi / 2$ and that the flag tip deflection cannot exceed 0.79 . Note that the tip deflection reaches the maximum feasible value of $w_{\text {tip }}=0.85$ and then starts to decrease as the inverted flag bends more (see Fig. 6(a)). Hence, it is impossible to explore the behaviour of the system at higher flow velocities based on the model proposed in Eq. (A.1).

Remarkably, one may use the proposed model given in Eq. (19) (that is the nonlinear equation of the motion in terms of the angle of rotation) while substituting sin and cos terms by their truncated Taylor expansions; this results in truncated equations in terms of the angle of rotation $\psi(x, t)$. In this case, a satisfactory convergence can be achieved with relatively high truncation order. Figure A.2 shows the convergence of tip rotation of the inverted flag with respect to the order of truncation. The solutions obtained via the proposed model given in Eq. (19) predict the deflection of the inverted flag correctly even for very large flow velocities where the flag is fully bent over one side. Indeed, the proposed model is suitable for very large deflections of the inverted flag and enables us to explore the existence of secondary bifurcations in the response of the system and to obtain the configuration of the system at high flow velocities.

\section{Appendix B. Functions of the nonlinear fluid-related force}

The functions required for the calculation of the normal force acting on the plate are presented here for the reader's convenience [5]:

$$
\begin{aligned}
\gamma(\vartheta, A) & =\frac{1}{g(R)+\cot \vartheta\left[f_{1}(\zeta(\vartheta))+f_{2}(\xi(\vartheta))-\vartheta f_{3}(\lambda(\vartheta))\right]} \\
f_{1}(\zeta) & =\frac{1}{2} \arctan \zeta-\arctan \left(\frac{\sin \left(\frac{1}{2} \arctan \zeta\right)}{\sqrt[4]{1+\zeta^{2}}+\cos \left(\frac{1}{2} \arctan \zeta\right)}\right)
\end{aligned}
$$




$$
\begin{aligned}
& f_{2}(\xi)=-\frac{1}{\xi}+\frac{1}{\xi} \frac{\cos \left(\frac{1}{2} \arctan v\right)}{\sqrt[4]{1+v^{2}}}+\frac{\sin \left(\frac{1}{2} \arctan v\right)}{\sqrt[4]{1+v^{2}}} \\
& f_{3}(\lambda)=\frac{1}{2} \mathrm{e}^{-\lambda} I_{1}(\lambda) \\
& g(R)=\frac{R}{2(R+2)}+\frac{1}{R+2} \sqrt{\frac{R}{R+2}} \frac{\sqrt{\frac{R}{R+2}}+2}{\left(\sqrt{\frac{R}{R+2}}+1\right)^{2}}+\frac{\ln 2}{2} \exp \left(\frac{-1.302}{R}\right) I_{1}\left(\frac{1.302}{R}\right)
\end{aligned}
$$

where $\zeta$, $\xi$, and $\lambda$ are defined as

$$
\zeta(\vartheta)=\frac{2 \sin \vartheta}{R}, \quad \xi(\vartheta)=\frac{2 \tan \vartheta}{R}, \quad \lambda(\vartheta)=-\frac{1}{R} \ln \left(\frac{\arctan (\sqrt{2} \tan \vartheta)}{\vartheta}-1\right),
$$

and $I_{1}$ is the modified Bessel function of the first kind and order 1.

\section{References}

[1] M. Amabili. Nonlinear vibrations and stability of shells and plates. Cambridge University Press, 2008.

[2] P. J. Attar, E. H. Dowell, and D. Tang. "Modeling aerodynamic nonlinearities for two aeroelastic configurations: delta wing and flapping flag". 44th AIAA/ASME/ASCE/AHS/ASC Structures, Structural Dynamics, and Materials Conference. 2003.

[3] T. Balint and A. Lucey. "Instability of a cantilevered flexible plate in viscous channel flow". Journal of Fluids and Structures 20.7 (2005), pp. 893-912.

[4] R. L. Bisplinghoff, H. Ashley, and R. L. Halfman. Aeroelasticity. Courier Corporation, 2013.

[5] W. Bollay. "A non-linear wing theory and its application to rectangular wings of small aspect ratio". ZAMM-Journal of Applied Mathematics and Mechanics/Zeitschrift für Angewandte Mathematik und Mechanik 19.1 (1939), pp. 21-35.

[6] P. Buchak, C. Eloy, and P. M. Reis. "The clapping book: wind-driven oscillations in a stack of elastic sheets". Physical review letters 105.19 (2010), 194301 (1-4).

[7] S. K. Datta and W. G. Gottenberg. "Instability of an elastic strip hanging in an airstream". Journal of Applied Mechanics 42.1 (1975), pp. 195-198.

[8] O. Doaré and S. Michelin. "Piezoelectric coupling in energy-harvesting fluttering flexible plates: linear stability analysis and conversion efficiency". Journal of Fluids and Structures 27.8 (2011), pp. 13571375.

[9] J. Dunnmon, S. Stanton, B. Mann, and E. H. Dowell. "Power extraction from aeroelastic limit cycle oscillations". Journal of Fluids and Structures 27.8 (2011), pp. 1182-1198.

[10] C. Eloy and L. Schouveiler. "Optimisation of two-dimensional undulatory swimming at high Reynolds number”. International Journal of Non-Linear Mechanics 46.4 (2011), pp. 568-576.

[11] C. Eloy, C. Souilliez, and L. Schouveiler. "Flutter of a rectangular plate". Journal of Fluids and Structures 23.6 (2007), pp. 904-919.

[12] C. Eloy, R. Lagrange, C. Souilliez, and L. Schouveiler. "Aeroelastic instability of cantilevered flexible plates in uniform flow". Journal of Fluid Mechanics 611 (2008), pp. 97-106.

[13] K. Gersten. "A nonlinear lifting-surface theory especially for low aspect-ratio wings". AIAA Journal 1.4 (1963), pp. 924-925.

[14] A. Giacomello and M. Porfiri. "Underwater energy harvesting from a heavy flag hosting ionic polymer metal composites". Journal of Applied Physics 109.8 (2011). 
[15] C. Guo and M. P. Païdoussis. "Stability of rectangular plates with free side-edges in two-dimensional inviscid channel flow". Journal of Applied Mechanics 67.1 (2000), pp. 171-176.

[16] P. Gurugubelli and R. Jaiman. "Self-induced flapping dynamics of a flexible inverted foil in a uniform flow”. Journal of Fluid Mechanics 781 (2015), pp. 657-694.

[17] L Huang. "Flutter of cantilevered plates in axial flow". Journal of Fluids and Structures 9.2 (1995), pp. 127-147.

[18] D. Kim, J. Cossé, C. Huertas Cerdeira, and M. Gharib. "Flapping dynamics of an inverted flag". Journal of Fluid Mechanics 736 (2013), R1 (1-12).

[19] A. Kornecki, E. H. Dowell, and J. E. O'Brien. "On the aeroelastic instability of two-dimensional panels in uniform incompressible flow". Journal of Sound and Vibration 47.2 (1976), pp. 163-178.

[20] S. Michelin and O. Doaré. "Energy harvesting efficiency of piezoelectric flags in axial flows". Journal of Fluid Mechanics 714 (2013), pp. 489-504.

[21] A. Nunn. Flapping of leaves and inverted-flags in a steady flow. Tech. rep. The University of Melbourne, 2017.

[22] S. Orrego, K. Shoele, A. Ruas, K. Doran, B. Caggiano, R. Mittal, and S. H. Kang. "Harvesting ambient wind energy with an inverted piezoelectric flag". Applied Energy 194 (2017), pp. 212-222.

[23] M. P. Païdoussis. "Plates in axial flow". Fluid-Structure Interactions: Slender Structures and Axial Flow. 2nd ed. Vol. 2. Oxford: Academic Press, 2016, pp. 539-641.

[24] E. C. Polhamus. "A concept of the vortex lift of sharp-edge delta wings based on a leading-edge-suction analogy". NASA technical note (1966).

[25] J. Ryu, S. G. Park, B. Kim, and H. J. Sung. "Flapping dynamics of an inverted flag in a uniform flow". Journal of Fluids and Structures 57 (2015), pp. 159-169.

[26] J. E. Sader, C. Huertas-Cerdeira, and M. Gharib. "Stability of slender inverted flags and rods in uniform steady flow". Journal of Fluid Mechanics 809 (2016), pp. 873-894.

[27] J. E. Sader, J. Cossé, D. Kim, B. Fan, and M. Gharib. "Large-amplitude flapping of an inverted flag in a uniform steady flow-a vortex-induced vibration”. Journal of Fluid Mechanics 793 (2016), pp. 524-555.

[28] C. Semler, G. Li, and M. P. Païdoussis. "The non-linear equations of motion of pipes conveying fluid". Journal of Sound and Vibration 169.5 (1994), pp. 577-599.

[29] M. J. Shelley, N. Vandenberghe, and J. Zhang. "Heavy flags undergo spontaneous oscillations in flowing water". Physical Review Letters 94.9 (2005), 094302 (1-4).

[30] K. Shoele and R. Mittal. "Flutter instability of a thin flexible plate in a channel". Journal of Fluid Mechanics 786 (2016), pp. 29-46.

[31] S. Taneda. "Waving motions of flags". Journal of the Physical Society of Japan 24.2 (1968), pp. 392-401.

[32] C. Tang, N.-S. Liu, and X.-Y. Lu. "Dynamics of an inverted flexible plate in a uniform flow". Physics of Fluids 27.7 (2015), 073601 (1-17).

[33] D. Tang, H Yamamoto, and E. H. Dowell. "Flutter and limit cycle oscillations of two-dimensional panels in three-dimensional axial flow". Journal of Fluids and Structures 17.2 (2003), pp. 225-242.

[34] L. Tang and M. P. Païdoussis. "A fluidelastic model for the dynamics of cantilevered plates with an additional spring support in axial flow". Computers and Structures 85.11-14 (2007), pp. 1089-1096.

[35] L. Tang and M. P. Païdoussis. "On the instability and the post-critical behaviour of two-dimensional cantilevered flexible plates in axial flow". Journal of Sound and Vibration 305.1-2 (2007), pp. 97-115.

[36] I. Wang, S. C. Gibbs, and E. H. Dowell. "Aeroelastic model of multisegmented folding wings: theory and experiment”. Journal of Aircraft 49.3 (2012), pp. 911-921. 
[37] Y. Watanabe, K Isogai, S Suzuki, and M Sugihara. “A theoretical study of paper flutter”. Journal of Fluids and Structures 16.4 (2002), pp. 543-560.

[38] T. Y.-T. Wu. "Hydromechanics of swimming propulsion. Part 1. Swimming of a two-dimensional flexible plate at variable forward speeds in an inviscid fluid". Journal of Fluid Mechanics 46.2 (1971), pp. 337-355.

[39] Y. Yadykin, V. Tenetov, and D. Levin. "The flow-induced vibration of a flexible strip hanging vertically in a parallel flow part 1: temporal aeroelastic instability". Journal of Fluids and Structures 15.8 (2001), pp. 1167-1185.

[40] N. Yamaguchi, T. Sekiguchi, K. Yokota, and Y. Tsujimoto. "Flutter limits and behavior of a flexible thin sheet in high-speed flow-II: experimental results and predicted behaviors for low mass ratios". Journal of Fluids Engineering 122.1 (2000), pp. 74-83.

[41] J. Zhang, S. Childress, A. J. Libchaber, and M. J. Shelley. "Flexible filaments in a flowing soap film as a model for one-dimensional flags in a two-dimensional wind". Nature 408.6814 (2000), pp. 835-839.

[42] W. Zhao, M. P. Païdoussis, L. Tang, M. Liu, and J. Jiang. "Theoretical and experimental investigations of the dynamics of cantilevered flexible plates subjected to axial flow". Journal of Sound and Vibration 331.3 (2012), pp. 575-587. 